\title{
Expression Pattern of Serum Cytokines in Hepatitis B Virus Infected Patients with Persistently Normal Alanine Aminotransferase Levels
}

\author{
Dengming He • Maoshi Li • Shimin Guo $\cdot$ Peng Zhu • \\ Hongfei Huang • Guohua Yan • Quanxin Wu • Shiqi Tao • \\ Zhaoxia Tan · Yuming Wang
}

Received: 25 February 2013 / Accepted: 26 July 2013 /Published online: 17 August 2013

(C) The Author(s) 2013. This article is published with open access at Springerlink.com

\begin{abstract}
Purpose About $60-80 \%$ of chronic hepatitis B virus (HBV) carriers are characterized with persistently normal alanine transaminase (ALT). Differences of cytokine expression are associated with the prognosis of HBV infection. We investigated the expression pattern of 30 cytokines associated with anti-HBV immunity in patients with normal ALT.

Methods Four patient groups (immune tolerance, inactive hepatitis B surface antigen carriers, resolved hepatitis B, and control; 10 subjects per group) were assigned. Thirty cytokines, including IFN- $\gamma$, IL-1 $\beta$, IL-2, IL-4, IL-6, IL-7, IL-9, IL-10, IL-12p40, IL-12p70, IL-15, IL-17A, IL-17C, IL-21, IL22, IL-23p19, IL-28A, IL-29, CCL5, CCL16, CCL20, CCL22, CXCL9, CXCL10, CXCL11, TNFRSF8, TNFRSF18, IL-6R, gp130, and TGF- $\beta 1$, were measured using a human cytokine antibody array. Signal intensities were obtained by laser scanner. Protein-protein interactions were analyzed by STRING (Search Tool for the Retrieval of Interacting Genes/Proteins). Results Significant differences of signal intensities were observed for IL-2, IL-4, IL-6, IL-7, IL-9, IL-10, IL-12p40, IL12p70, IL-15, IL-21, IL-23p19, IL-28A, and IL-29. The lowest intensity was in controls. Among three HBV infection groups, significant differences were observed in IL-2, IL-4,
\end{abstract}

Dengming He and Maoshi Li contributed equally to this work.

Electronic supplementary material The online version of this article (doi:10.1007/s10875-013-9931-0) contains supplementary material, which is available to authorized users.

D. He $\cdot \mathrm{M} . \mathrm{Li} \cdot \mathrm{S}$. Guo $\cdot$ P. Zhu $\cdot$ H. Huang $\cdot$ G. Yan $\cdot$ Q. Wu $\cdot$

S. Tao $\cdot$ Z. Tan $\cdot$ Y. Wang $(\triangle)$

Institute of Infectious Disease, Southwest Hospital,

Third Military Medical University, Chongqing, China

e-mail:wym417@163.com

D. $\mathrm{He}$

Liver Disease Diagnoses and Treatment Center of Chinese PLA,

The 88th Hospital of Chinese PLA, Tai'an, China
IL-12p70, IL-15, IL-21, IL-23p19, and IL-29. The highest intensity was in the inactive group. All cytokines with significant differences were involved JAK-STAT signaling that upregulate FOXP3, SOCS3 and MX1.

Conclusion Differential expression of cytokines in JAK-STAT signaling is an important factor associated with prognosis of HBV infection. The elevation of $\gamma \mathrm{C}$ cytokines, IL-12p70, IL23p19, and IL-29 may promote spontaneous HBeAg seroconversion and $\mathrm{HBV}$ clearance.

Keywords Protein array $\cdot$ hepatitis B virus $\cdot$ alanine transaminase $\cdot$ cytokine $\cdot$ chemokine

\section{Introduction}

An estimated 350 million individuals worldwide are chronic hepatitis B surface antigen (HBsAg) carriers, mainly infected by vertical transmission of hepatitis B virus (HBV) from mother to neonate $[1,2]$. Although HBsAg carriers are at increased risk of developing cirrhosis, hepatic decompensation and hepatocellular carcinoma, most carriers will not develop hepatic complications from chronic hepatitis B during their lifetime. About $60-80 \%$ of $\mathrm{HBsAg}$ carriers are characterized with persistently normal alanine transaminase (ALT) levels [1-3], and during HBV infection the persistently normal ALT levels are a fundamental character of the immune tolerance phase, inactive HBsAg carrier phase, and resolved hepatitis B phase $[1,3,4]$. However, patients in the inactive HBsAg carrier phase and resolved hepatitis B phase with lower levels of HBsAg or HBsAg loss, respectively [5-8], have a better prognosis than patients with active hepatitis characterized by ALT elevation.

Differences in anti-HBV immunity are associated with the prognosis of HBV infection [9]. During chronic liver damage, adaptive immune cells are crucially involved in the pathogenesis of hepatic inflammation; particularly $\mathrm{T}$ cells play important 
roles in antiviral defense [9]. The activity of T lymphocytes is largely dependent on the differentiation of naive T cells into Thelper 1 (Th1), Th2, Th9, Th17, and Th22 effector T-cell subsets via the release of cytokines and other cofactors. Inflammatory chemokines can recruit monocytes, neutrophils and other effector cells from the blood to sites of infection or tissue damage. Certain inflammatory chemokines activate immune cells to initiate an immune response or promote wound healing [10]; they are released by many different cell types and activate cells of both the innate and adaptive immune system. Interactions between cytokines/chemokines and the cellular immune system is a dynamic process [11]. The interactions of positive and negative stimuli, as well as positive and negative regulatory loops are complex and often involve multiple cytokines.

In homeostasis, the liver exerts various metabolic functions and serves as a central "immunological" organ [12-14]. The pattern of cytokines and chemokine present in serum was linked to HBV viral load and was consistent with the degree of inflammation during chronic hepatitis [9]. To observe the expression pattern of cytokines/chemokines and differences in immunity during different phases of HBV infection with persistently normal ALT levels, a protein array was used to detect 30 cytokines and chemokines associated with cellular immunology, such as T-cell proliferation and activation, antiviral immunity, immunoregulatory activity, inflammatory response, chemotactic activity for lymphocytes and monocytes.

\section{Subjects and Methods}

\section{Subjects}

HBV carrier subjects in the immune tolerance phase, inactive HBsAg carrier phase, or resolved hepatitis B phase were recruited from the Department of Infectious Diseases, Southwest Hospital, Chongqing, China. For the immune tolerance phase and inactive HBsAg carrier phase, inclusion criteria were as follows: a) a history of being HBsAg positive over 10 years; b) persistently normal ALT levels (upper limit of normal is 30 $\mathrm{U} / \mathrm{L}$ for men and $19 \mathrm{U} / \mathrm{L}$ for women) over 2 years confirmed by monitoring at 3 to 6 months intervals; c) HBV DNA levels over $1 \times 10^{6} \mathrm{IU} / \mathrm{mL}$ for immune tolerance phase and undetectable for inactive HBsAg carrier phase; d) HBsAg levels less than $250 \mathrm{IU} / \mathrm{mL}$ for inactive HBsAg carrier phase.

For the resolved hepatitis $\mathrm{B}$ phase, inclusion criteria were as follows: a) anti-HBs, anti-HBe or anti-HBc positive; b) $\mathrm{HBsAg}$ negative; c) undetectable serum HBV DNA levels; d) normal ALT levels.

Control subjects were recruited from healthy blood donors. Inclusion criteria were as follows: a) HBsAg, anti-HBs, $\mathrm{HBeAg}$, anti-HBe or anti-HBc negative; b) undetectable serum HBV DNA levels; c) normal ALT levels.
Exclusion criteria were as follows: a) receiving interferon (IFN) or nucleoside analogues antiviral therapy in the last 2 years; b) a history of fever in the past 6 months; c) clinical and/or biochemical signs of chronic active hepatitis (spider angiomas, liver palm, splenomegaly, decreased serum albumin, or portal hypertension); d) co-infection with hepatitis $\mathrm{C}$ virus, hepatitis $\mathrm{D}$ virus or human immunodeficiency virus; e) coexisting serious medical or psychiatric illness; f) organ or bone marrow transplantation; g) recent treatment with systemic corticosteroids, immunosuppressants, or chemotherapeutic agents; h) a serum alpha-fetoprotein levels over $50 \mathrm{ng} / \mathrm{mL}$.

Written informed consent was obtained from each patient for his or her participation in the study. The study protocol conformed to the ethical guidelines of the 1975 Declaration of Helsinki as reflected in a priori approval by the ethics committee of the Southwest Hospital.

\section{Serological Assays}

Routine biochemical tests were performed using automated techniques. $\mathrm{HBsAg}$, anti-HBs, $\mathrm{HBeAg}$, anti-HBe, anti-HBc, and alpha-fetoprotein levels were detected by Electrochemiluminescence (Architect, Abbott Laboratories, Abbott Park, IL, USA). Antibodies to hepatitis $\mathrm{C}$ virus, hepatitis $\mathrm{D}$ virus and human immunodeficiency virus were detected by routine, commercially available enzyme immunoassays (KHB, Shanghai, China). Serum HBV DNA levels were quantified using a commercially available polymerase chain reaction (PCR) assay (LightCycler ${ }^{\circledR} 480$ Real-Time PCR System, Roche, Basel, Switzerland) with a lower limit of quantification of $200 \mathrm{IU} / \mathrm{ml}$.

\section{Serum Samples}

Serum samples were obtained by centrifugation of clotted blood at 3,000 rpm for $5 \mathrm{~min}$. Serum samples were immediately stored at $-80{ }^{\circ} \mathrm{C}$ and thawed prior to analysis.

\section{Cytokines and Chemokines Selected for Protein Array}

A total of 30 cytokines and chemokines, including IFN $-\gamma$, interleukin-1 $\beta$ (IL-1 $\beta$ ), IL-2, IL-4, IL-6, IL-7, IL-9, IL-10, IL-12p40, IL-12p70, IL-15, IL-17A, IL-17C, IL-21, IL-22,


chemokine ligand 5 (CCL5), CCL16, CCL20, CCL22, CXC-chemokine ligand 9 (CXCL9), CXCL10, CXCL11, tumor necrosis factor receptor superfamily member 8 (TNFRSF8, CD30), TNFRSF18 (glucocorticoid-induced TNFR-Related protein, GITR), IL-6 receptor (IL-6R), IL6 signal transducer (IL-6ST, gp130), and transforming growth factor $\beta 1$ (TGF- $\beta 1$ ), were selected to be measured in a protein array to demonstrate different aspects of innate, adaptive and chemokine responses to HBV exposure. For 
example, Th1 activation is indicated by IFN- $\gamma$, IL-12, IL-2, IL-15 and IL-7; Th2 activation is indicated by IL-10, IL-4, and TNFRSF8; Th17 activation is indicated by IL-23, IL-1 $\beta$, IL17, and IL-21; Th9 and Th22 activation is indicated by IL-9 and IL-22; regulatory T cell (Treg) activation is indicated by TNFRSF18, TGF- $\beta 1$; and innate immune responses might result in IL-6, IL-6R, IL-6ST, IL-29, and IL-28A.

\section{Cytokines and Chemokines Analyses}

Cytokines and chemokines were measured in stored serum using a designed human cytokine antibody array (RayBiotech, Inc, GA, USA) according to the manufacturer's instructions. Images were captured using a laser scanner (Axon GenePix 4000B, Molecular Device, CA, USA) using the "green" channel (excitation frequency $=532 \mathrm{~nm}$ ). The signal intensities obtained from the laser scanner. Locating signal intensities to the antibody array map, mean signal intensities, subtraction of the background, and normalization of data from different samples were achieved by the analysis tool.

\section{Protein-Protein Interactions}

Protein-protein interactions were analyzed by STRING (Search Tool for the Retrieval of Interacting Genes/Proteins), a database of known and predicted protein interactions [15]. The pathway was analyzed by KEGG (Kyoto Encyclopedia of Genes and Genomes).

\section{Statistical Analysis}

Signal intensities were expressed as mean \pm SD (range). Statistical analysis was tested using analysis of variance (ANOVA). All tests for significance and resulting $\mathrm{p}$ values were two-sided, with a level of significance of 0.05 . The statistical software used for this analysis was SPSS 18.0.

\section{Results}

\section{Study Subjects}

A total of 40 subjects were studied consisting of 10 subjects of immune tolerance phase (tolerance group), 10 subjects of inactive HBsAg carrier phase (inactive group), 10 subjects of resolved hepatitis B phase (resolved group), and 10 healthy controls (control group). The demographic data and characteristics of HBV infection of the subjects are shown in Table I.

Protein-Protein Interactions

According to KEGG, all 30 cytokines and chemokines were included in the cytokine-cytokine receptor interaction pathway $\left(p=5.449 \times 10^{-39}\right)$. In addition, 17 cytokines and eight cytokines chemokines were included in JAK-STAT signaling pathway $\left(p=3.60 \times 10^{-18}\right)$ and Toll-like receptor (TLR) signaling pathway $\left(p=1.97 \times 10^{-6}\right)$, respectively.

Actions of these cytokines and chemokines are shown in Fig. 1. Of these cytokines and chemokines, there were 234 interactions over medium confidence (0.400), 181 interactions over high confidence $(0.700)$ and 114 interactions over highest confidence (0.900) (Supplementary Table 1).

Increased Expression of Cytokines in HBV Infection Groups Compared with Controls

Four typical images of the protein array captured using a laser scanner are shown in Fig. 2. Among 30 cytokines and chemokines, significant differences of signal intensities among four groups were observed in 13 cytokines, including IL-2 $\left(p=3.25 \times 10^{-5}\right)$, IL-4 $\left(p=3.49 \times 10^{-4}\right)$, IL-6 $(p=0.006)$, IL-7 $(p=0.013)$, IL-9 $(p=0.004)$, IL-10 $(p=0.022)$, IL-12p 40 $(p=0.025), \mathrm{IL}-12 \mathrm{p} 70\left(p=1.215 \times 10^{-4}\right), \mathrm{IL}-15(p=0.004), \mathrm{IL}-$ 21 ( $p=0.013)$, IL-23p19 ( $p=0.013)$, IL-28A ( $p=0.004)$, and IL$29\left(p=2.104 \times 10^{-4}\right)$ (Fig. 3).

The lowest expression of these 13 cytokines was seen in the control group. Significant differences between control and inactive groups were observed in all 13 cytokines. Significant differences between control and tolerance groups was observed in IL-2 $(p=0.005)$ and IL-29 $(p=0.028)$. Significant differences between control and resolved groups was observed for IL-2 $(p=0.002)$, IL-6 $(p=0.001)$, IL-7 $(p=0.002)$, IL-9 $\left(p=4.38 \times 10^{-4}\right)$, IL-10 $(p=0.005)$, IL-12 p40 $(p=0.033)$, IL-12 p70 $(p=0.023)$, IL-23 p19 $(p=0.022)$, IL-28A $\left(p=3.66 \times 10^{-4}\right)$, and IL-29 $\left(p=7.88 \times 10^{-5}\right)$, but not IL-4, IL15, and IL-21 (Fig. 3).

\section{Differential Expression of Cytokines in HBV Infection Groups}

Among the three $\mathrm{HBV}$ infected groups, significant differences were observed in seven cytokines, including IL-2 $(p=0.009)$, IL-4 ( $p=0.001)$, IL-12p70 ( $p=0.001)$, IL-15 ( $p=0.003)$, IL-21 $(p=0.015)$, IL-23p19 $(p=0.007)$, and IL-29 $(p=0.038)$ (Fig. 4). Significant differences between the tolerance and inactive groups were observed for all seven cytokines, including IL-2 $(p=0.004)$, IL-4 $\left(p=4.55 \times 10^{-4}\right)$, IL-12p70 $\left(p=3.94 \times 10^{-4}\right)$, IL-15 $(p=0.001)$, IL-21 $(p=0.009)$, IL$23 p 19(p=0.002)$, and IL-29 $(p=0.033)$. Significant differences between the inactive and resolved groups were observed for six cytokines besides IL-29, including IL-2 $(p=0.013)$, IL4 ( $p=0.007), \mathrm{IL}-12 \mathrm{p} 70(p=0.014), \mathrm{IL}-15$ ( $p=0.049), \mathrm{IL}-21$ $(p=0.015)$, and IL-23p19 $(p=0.023)$. Significant differences between the resolved and tolerance groups were only observed for IL-29 $(p=0.021)$. Noticeably, the highest intensities of these seven cytokines were observed in the inactive group. 
Table I Demographic data and subject characteristics

${ }^{a}$ The upper limits of normal are $30 \mathrm{U} / \mathrm{L}$ for men and $19 \mathrm{U} / \mathrm{L}$ for women

\begin{tabular}{lllll}
\hline Characteristic & Tolerance $(n=10)$ & Inactive $(n=10)$ & Resolved $(n=10)$ & Control $(n=10)$ \\
\hline Male-no. (\%) & $5(50)$ & $5(50)$ & $5(50)$ & $5(50)$ \\
Age (years) & $29 \pm 9$ & $30 \pm 7$ & $33 \pm 8$ & $24 \pm 6$ \\
Total Bilirubin $(\mu \mathrm{mol} / \mathrm{L})$ & $11.6 \pm 2.4$ & $10.6 \pm 2.4$ & $10.5 \pm 2.7$ & $11.2 \pm 1.3$ \\
ALB $(\mathrm{g} / \mathrm{L})$ & $47 \pm 2.5$ & $47 \pm 3.3$ & $46 \pm 2.7$ & $49 \pm 2.9$ \\
ALT $(\mathrm{U} / \mathrm{L})^{\mathrm{a}}$ & $20 \pm 5$ & $14 \pm 4$ & $16 \pm 5$ & $13 \pm 6$ \\
HBV DNA & $7.0 \pm 0.3$ & Undetectable & Undetectable & Undetectable \\
$(\log 10 \mathrm{IU} / \mathrm{mL})$ & & & \\
\hline
\end{tabular}

Protein-Protein Interactions of Cytokines with Significant Differences

All cytokines with significant differences were involved in JAK-STAT signaling $\left(p=5.080 \times 10^{-15}\right)$. As IL-2, IL-4, IL-15, and IL-21 belong to the common $\gamma$ receptor chain $(\gamma \mathrm{C})$ family of cytokines, we analyzed the actions of these cytokines and the top ten confidence proteins (Fig. 5a). This action network acts as to up-regulate FOXP3 expression. IL-12p40, IL$12 \mathrm{p} 70$, and IL-23p19 are members of the IL-12 family. The actions of IL-12p40, IL-12p70, IL-23p19 and the top ten confidence proteins act to up-regulate the expression of suppressor of cytokine signaling 3 (SOCS3), a member of the STAT-induced STAT inhibitor (Fig. 5b). The similar upregulating expression of SOCS3 was observed for IL-6 and the top 10 confidence proteins (Fig. 5c). Furthermore, IL-29 is associated with TLR pathways and acts as an up-regulating interferon-induced GTP-binding protein MX1 in the actions network of IL-29 and the top 10 confidence proteins (Fig. 5d). Interactions of IL-2, IL-4, IL-15, IL-21 and the top 10 confidence proteins, IL-12p40, IL-12p35, IL-23p19 and the top 10 confidence proteins, IL-6 and the top 10 confidence cytokines, and IL-29 and the top 10 confidence cytokines are shown in Supplementary Tables 2, 3, 4, and 5 .
Fig. 1 Actions of 30 cytokines and chemokines in serum from HBV carriers. The edges represent the actions: blue line binding action; black line reaction action; yellow line expression action. Direction of the action is indicated by the symbol at the end of the edge next to the protein that is acted upon. Down-Regulation is represented by a red bar and upregulation by a green arrow. A yellow circle describes that the directionality of the interaction is known, but the result of the interaction is not known. IL1B IL1 $\beta$; IL2 IL-2; IL4 IL-4; IL6 IL-6; IL6R IL-6 receptor; IL6ST IL-6 signal transducer; IL7 IL-7; IL9 IL-9; IL10 IL-10; IL12A IL12p35; IL12B IL-12p40; IL15 IL-15; IL17A IL-17A; IL17C IL-17C; IL21 IL-21; IL22 IL-22; IL23A IL-23p19; IL28A IL-28A; IL29 IL-29; IFNG IFN- $\gamma$; TNFRSF8 tumor necrosis factor receptor superfamily, member 8 ; TNFRSF 18 tumor necrosis factor receptor superfamily, member 18 ; TGFB1 TGF- $\beta 1$

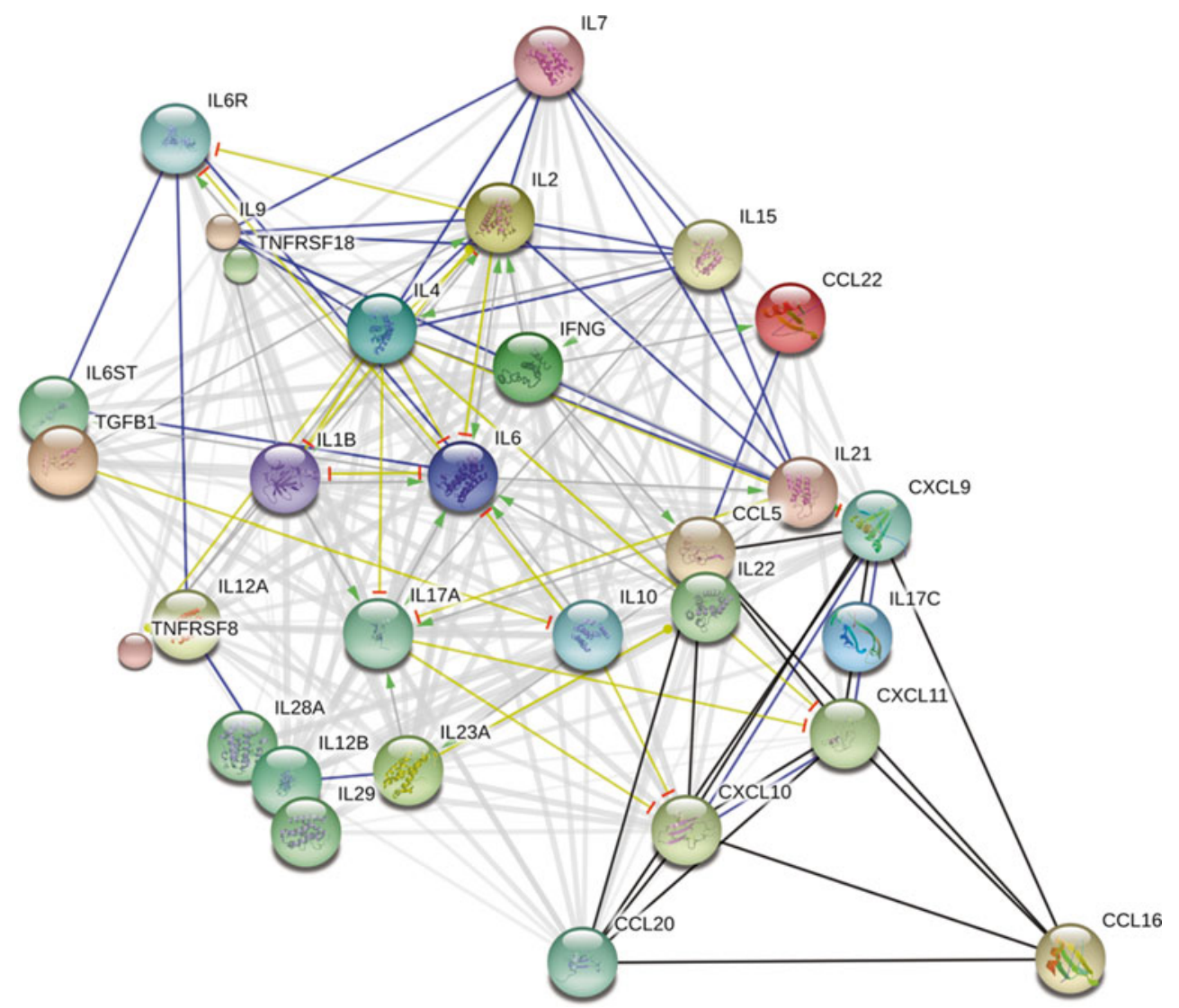




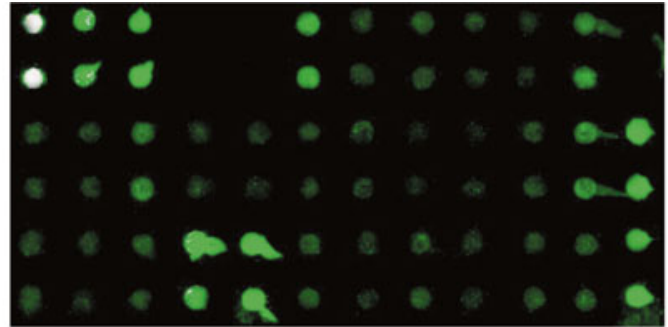

Control

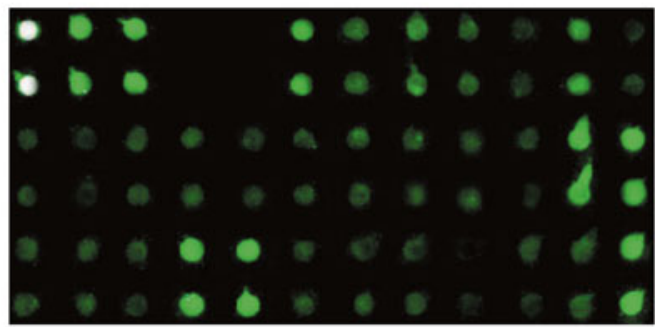

Tolerance

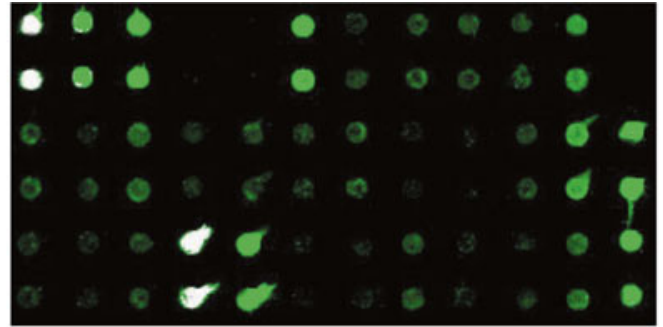

Inactive

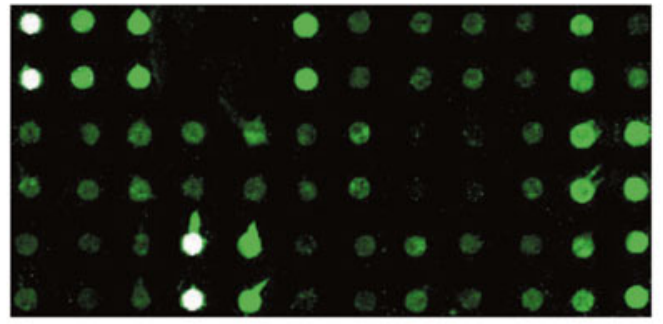

Resolved

\begin{tabular}{|c|c|c|c|c|c|c|c|c|c|c|c|c|}
\hline & A & $\mathrm{B}$ & C & D & $E$ & $\mathrm{~F}$ & G & $\mathrm{H}$ & 1 & $\mathrm{~J}$ & $\mathrm{~K}$ & $\mathrm{~L}$ \\
\hline 1 & POS & POS & POS & NEG & NEG & CCL16 & TNFRSF 18 & IL15 & IL7 & IL29 & CXCL10 & CCL2O \\
\hline 2 & POS & POS & POS & NEG & NEG & $\mathrm{CCL} 16$ & TNFRSF 18 & IL15 & IL7 & IL29 & CXCL10 & $\mathrm{CCL} 20$ \\
\hline 3 & IFNG & IL17A & IL21 & IL4 & CXCL11 & CCL22 & IL10 & IL17C & IL22 & IL6 & CXCL9 & CCL5 \\
\hline 4 & IFNG & IL17A & IL21 & IL4 & CXCL11 & $\mathrm{CCL} 22$ & IL 10 & IL17C & IL22 & IL6 & CXCL9 & CCL5 \\
\hline 5 & IL12B & IL1B & IL23A & IL6R & IL6ST & TNFRSF8 & IL 12p70 & IL2 & IL28A & IL9 & TGFB1 & POS \\
\hline 6 & IL12B & IL1B & IL23A & IL6R & IL6ST & TNFRSF8 & IL.12p70 & IL2 & IL28A & IL9 & TGFB1 & POS \\
\hline
\end{tabular}

Fig. 2 Representative images of the protein array captured using a laser scanner. Control control group; tolerance immune tolerance group; inactive inactive HBsAg carrier; Resolved resolved hepatitis B

\section{Discussion}

Apart from the immune active phase (chronic hepatitis B), persistently normal ALT levels are a basic feature in HBV infection [3]. The immune response initiated by the T-cell response to viral antigens is thought to be fundamental for viral clearance and disease pathogenesis in HBV infection [16] and the recognition of infected hepatocytes by HBVspecific CD8 cells is assumed to be the central mechanism causing both liver damage and promoting virus control. HBV replication can be inhibited independent of liver damage in the presence of an effective HBV-specific CD8 response [17]. Cytokines and chemokines play a crucial role in initiating, maintaining, and regulating immunological homeostatic and inflammatory processes. Indeed, measuring the cytokine and chemokine profiles in patients provides a useful indication on disease status. Inflammatory responses during liver injury comprise resident and infiltrating immune cells. Noncytolytic intracellular viral inactivation by cytokines released by virus-inactivated lymphomononuclear cells could have an important role in the clearance of HBV without killing infected cells [16]. Therefore, we hypothesized that the expression pattern of serum cytokines and chemokines might be associated with different phases of HBV infection. While several of the cytokines selected are pleiotropic and secreted by both adaptive and innate immune cells, the overall pattern of cytokine production was used to determine the predominant arm of the immune response thought to be activated.

Among 13 cytokines with significant differences of expression, IL-2, IL-4, IL-7, IL-9, IL-15, and IL-21 belong to $\gamma \mathrm{C}$ cytokines, which signal through specific receptor complexes sharing $\gamma \mathrm{C}$. Because of this shared receptor usage, $\gamma \mathrm{C}$ cytokines share similar functions while each also possesses unique features. Predominant functions relate to the critical regulation of lymphocyte development, homeostasis, and functions [18]. Both IL-7 and IL-9 showed no significant difference among HBV infection group. The highest expression of IL-2, IL-4, IL-15, and IL-21 were observed in inactive HBsAg carriers. Although sharing common $\gamma \mathrm{C}$, the role of IL-2, IL-4, IL-15, and IL-21 in the regulation of lymphocytes is markedly different. IL-2 is the quintessential growth factor for T cells and theoretically would be ideal for expanding $\mathrm{T}$ cells. Besides stimulating effector T cells, IL-2 also mediates the development and homeostasis of Tregs [18]. IL-4 acts as a growth factor for Th2 cells and a co-stimulator of DNA-synthesis and induces the expression of class II MHC molecules on resting 

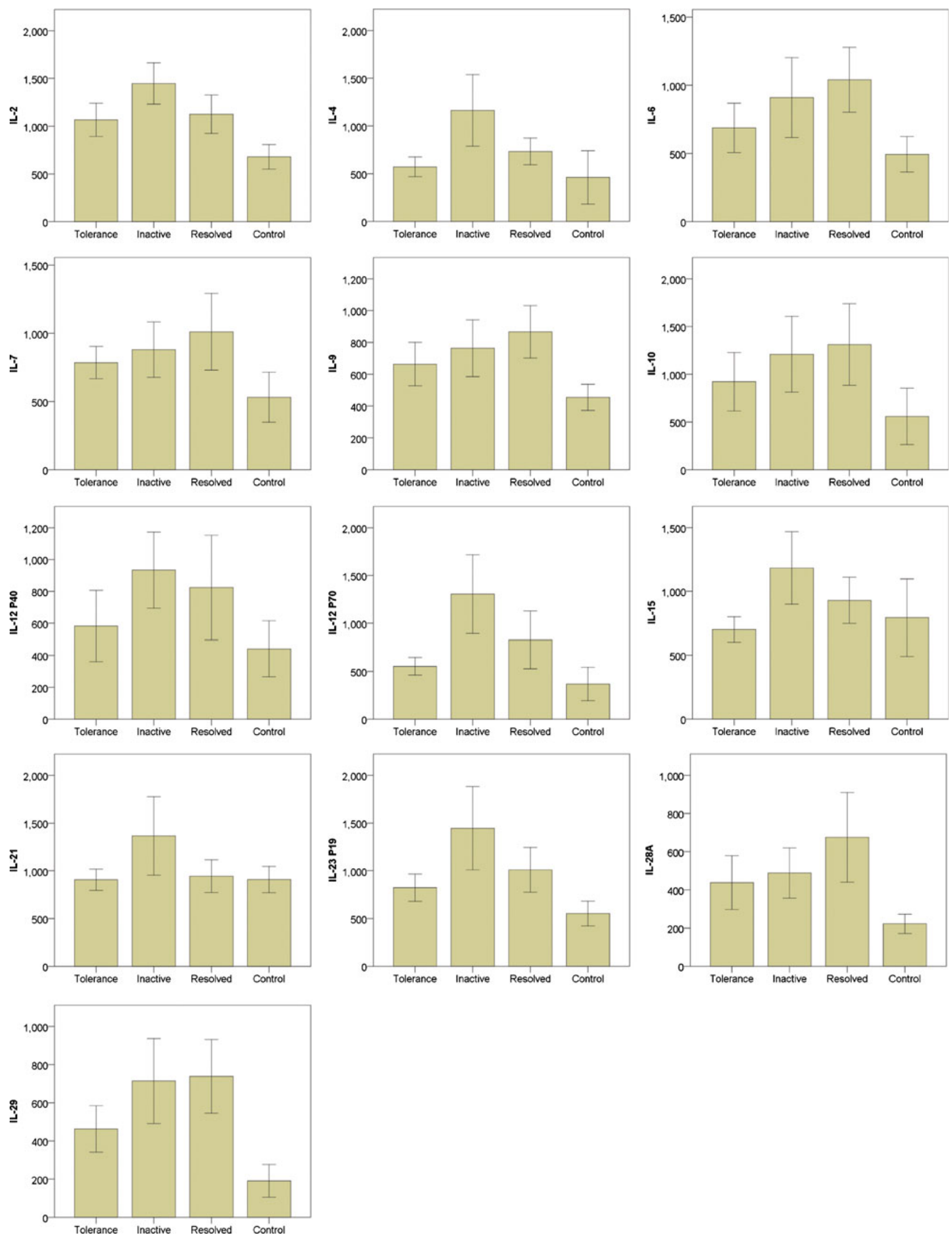

Fig. 3 Signal intensities of 13 cytokines with significant differences among the tolerance, inactive, resolved, and control groups. Control control group; tolerance immune tolerance group; inactive inactive HBsAg carrier group; Resolved resolved hepatitis B group 

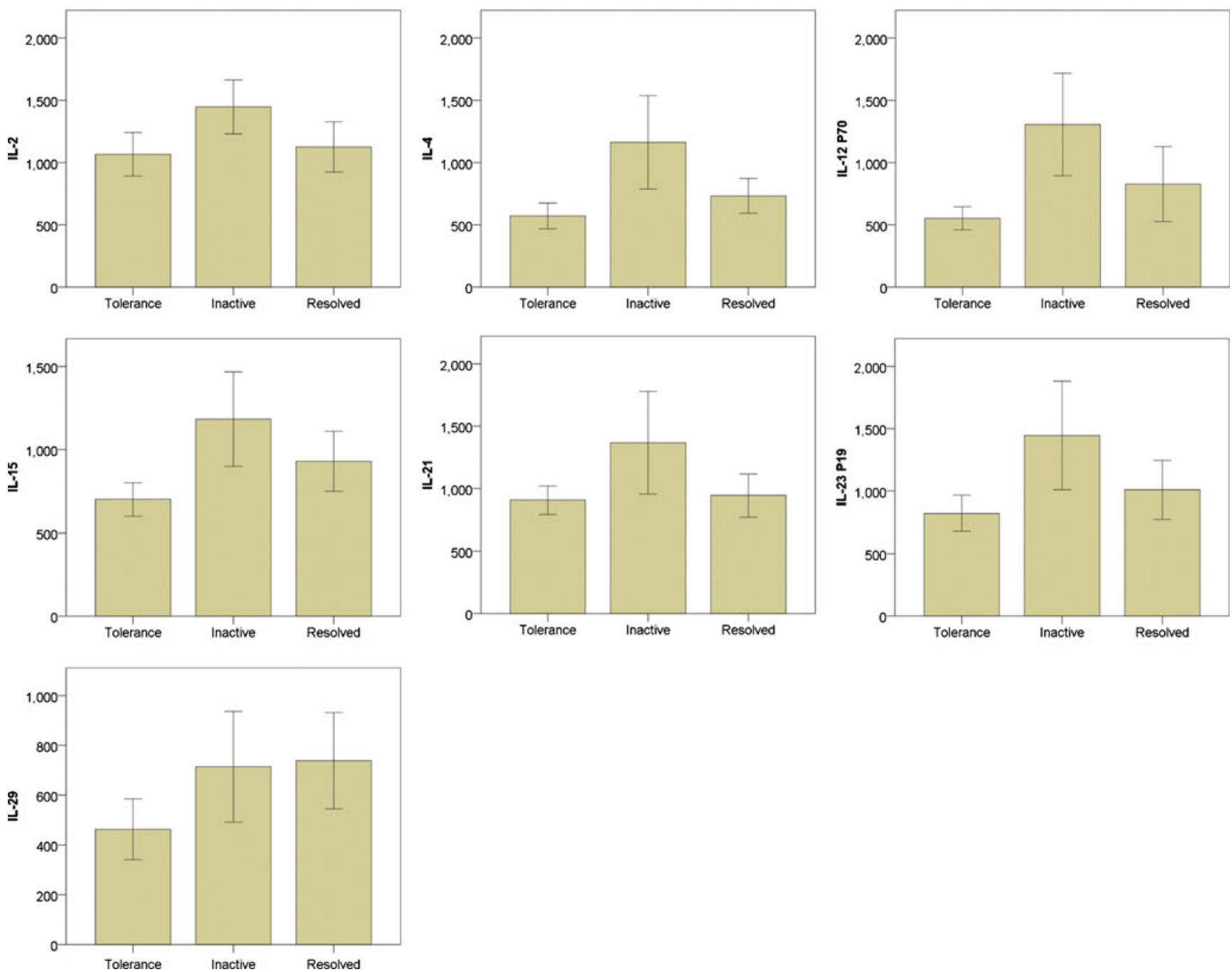

Fig. 4 Signal intensities of seven cytokines with significant differences among the tolerance, inactive, and resolved groups. Control control group; tolerance immune tolerance group; inactive inactive HBsAg carrier group; Resolved resolved hepatitis B group

B-cells [19-21]. Although sharing many functions with IL-2, IL-15 has distinct functions in vivo. In mouse studies, IL-15 was important for the development and homeostasis of memory CD8 T cells, NK cells, NKT cells, and certain subsets of intestinal CD8 T cells. In a C57BL/6 mouse model, IL-15 suppressed HBV replication in an IFN- $\beta$-dependent manner and exerted anti-HBV functions independent of the common IL-2 $\gamma(\mathrm{c}) \mathrm{R}[22,23]$. IL-21 is primarily produced by CD4 T cells, particularly Th17 CD4 T cells and NKT cells. Its expression is up-regulated upon activation. However, it does not appear to play a major role in the development of immune cells, as IL-21R deficient mice do not display major immune cell deficiencies [24, 25]. High serum IL-21 levels after 12 weeks of antiviral therapy can predict $\mathrm{HBeAg}$ seroconversion in chronic hepatitis B [26]. A recent study showed that the $\gamma$ C cytokines IL-2, IL-7, IL-15, and IL-21 but not IL-4 induced Tim-3 expression on naive, effector, and memory subsets of $\mathrm{T}$ cells [27]. According to the action network, IL-2, IL-4, IL-15, and IL-21 up-regulate FOXP3 expression, which functions as a master regulator in the development and function of Tregs.

IL-12p40, IL-12p70, and IL-23p19, which showed significant differences, belong to the IL-12 family of heterodimeric cytokines. The $\mathrm{p} 40$ chain can pair with $\mathrm{p} 35$ or $\mathrm{p} 19$ to form IL12 p70 or IL-23, which are mainly proinflammatory and prostimulatory cytokines with key roles in the development of the Th1 and Th17 subsets of helper T cells, respectively [28, 29]. Serum levels and hepatic expression of IL-23 were significantly up-regulation in chronic hepatitis $B$ patients and the molecular mechanism for HBV-induced IL-23 expression involved activation of the ERK/NF-KB pathway by HBV $\mathrm{x}$ protein [30]. Nevertheless, there was no significant difference in the expression of IFN- $\gamma$ and IL-17A, the effector cytokines of Th1 and Th17 subsets, respectively. Elevation of IL-12p70 and IL-23p19 in HBV infected patients suggests they might promote Th differentiation rather than a significant difference of Th subsets when ALT levels are persistently normal. IL-6, 

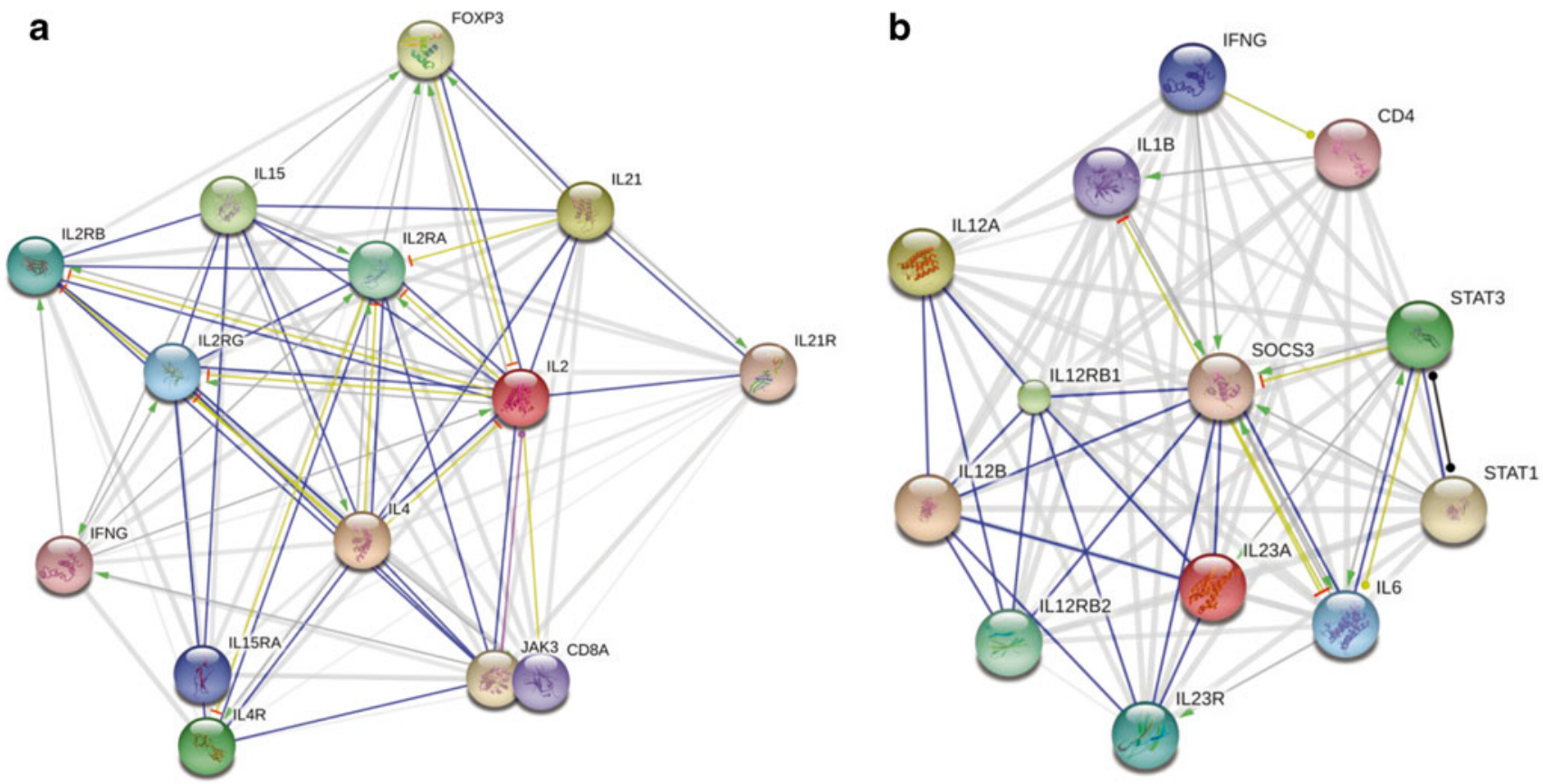

C

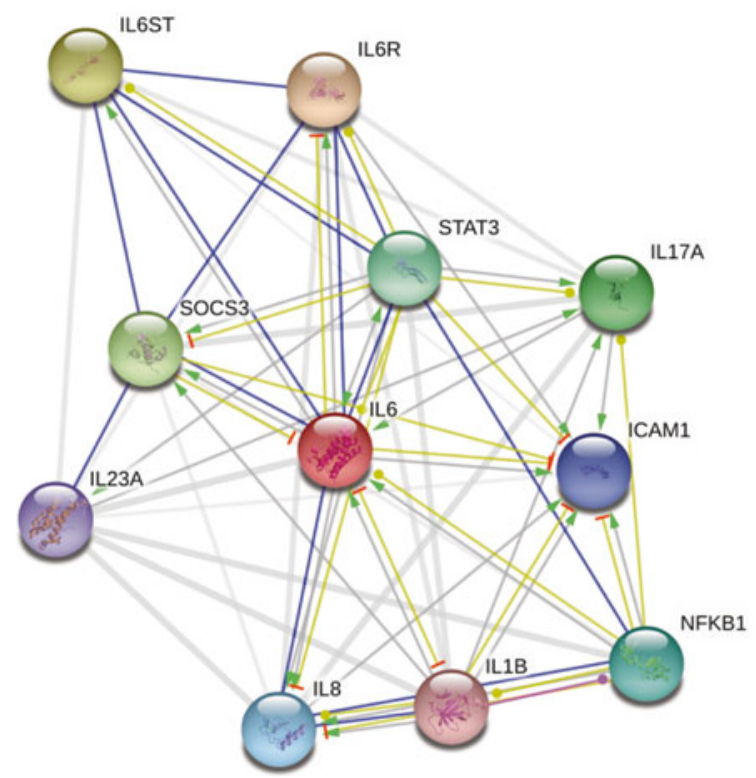

Fig. 5 Action of significant difference cytokines. a Actions of IL-2, IL-4, IL-15, IL-21, and the top 10 confidence proteins. b Actions of IL-12p40, IL-12p35, IL-23p19, and the top 10 confidence proteins. c Actions of IL6 and the top 10 confidence proteins. d Actions of IL-29 and the top 10 confidence proteins. The edges represent the actions: blue line binding action; black line reaction action; yellow line expression action; purple line catalysis action. Direction of the action is indicated by the symbol at the end of the edge next to the protein that is acted upon. Down-Regulation is represented by a red bar and up-regulation by a green arrow. A circle describes that the directionality of the interaction is known, but the result of the interaction is not known. IL2 IL-2; IL2RA IL-2 receptor $\alpha$; IL2RB IL-2 receptor $\beta$; IL2RG IL-2 receptor $\gamma$; IL4 IL-4; IL4R IL-4 receptor; IL15 IL-15; IL15RA IL-15 receptor $\alpha$; IL21 IL-21; IL21R IL-21 d

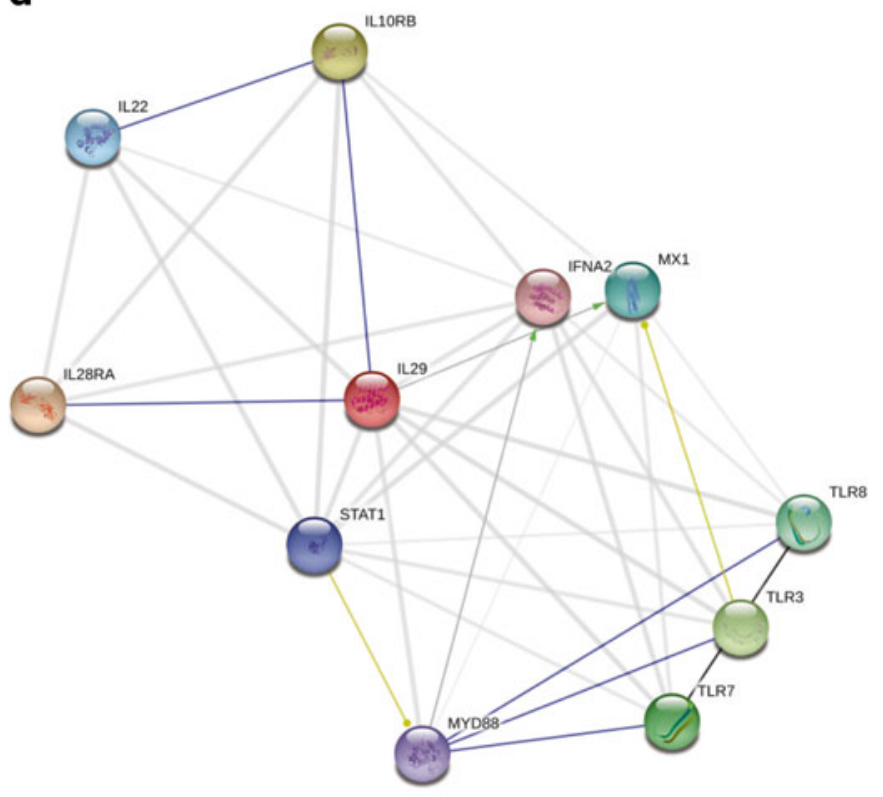

receptor; IFNG IFN- $\gamma$; JAK3 Janus kinase 3; CD8A CD8a molecule; FOXP3 Forkhead box P3; IL6 IL-6; IL12A IL-12p35; IL12B IL-12p40; IL23A IL-23p19; IL12RB1 IL-12 receptor $\beta 1$; IL12RB2 IL-12 receptor $\beta 2$; IL23R IL-23 receptor; SOCS3 suppressor of cytokine signaling 3; ILIB IL-1 $\beta$; CD4 CD4 molecule; STAT1 signal transducer and activator of transcription 1; STAT3 signal transducer and activator of transcription 3; IL6R IL-6 receptor; IL6ST IL-6 signal transducer; IL17A IL-17A; IL8 IL-8; NFKB1 nuclear factor of kappa light polypeptide gene enhancer in B-cells 1; ICAM1 intercellular adhesion molecule 1; IL29 IL-29; IFNA2 IFN- $\alpha 2 ; M X 1$ myxovirus resistance 1 ; IL1ORB IL-10 receptor $\beta$; IL22 IL22; IL28RA IL-28 receptor $\alpha$; MYD88 myeloid differentiation primary response gene (88); TLR7 toll-like receptor 7; TLR3 toll-like receptor 3; TLR 8 toll-like receptor 8 
which has hepatoprotective effects [31], showed a significant difference between control and HBV infection groups. A recent study demonstrated IL-6 controlled HBV replication at transcription [32]. SOCS3, which has extensive interactions with IL-12p40, IL-12p35, IL-23p19, and IL-6, is a cytokineinducible negative regulator of cytokine signaling and can inhibit the activity of JAK2 kinase. Thus, negative regulation might play an important role in maintaining the stability of HBV infection or normal ALT levels.

IL-29 (IFN- $\lambda 1$ ), a cytokine with immunomodulatory activity, may play a role in antiviral immunity and up-regulate MHC class I antigen expression [33, 34]. Although it belongs to the JAK-STAT signaling pathway, IL-29 is linked closely with TLR signaling pathways according to the interaction network and up-regulates MX1 expression, a key component of the antiviral state induced by IFNs, which inhibits HBV replication at a posttranscriptional level [35-37]. The elevation of IL-29 in the inactive and resolved groups, suggested that IL-29 plays an important role in anti-HBV immunity, especially compared with IFN- $\gamma$.

Immune tolerance phase is the first phase of chronic HBV infection, characterized by HBeAg-positive, high serum HBV DNA levels, and normal ALT levels. Compared with controls, significant differences in cytokine expression only were observed for IL-2 and IL-29. Thus, HBV infection during the immune tolerance phase may have little influence on the expression pattern of cytokines. However, this result reflects the key immune role of IL-2 and IL-29 during HBV infection.

Since $\gamma C$ cytokines, IL-12p70, IL-23p19, and IL-29 were elevated in the inactive HBsAg carrier and resolved hepatitis B groups, they may be potential biomarkers to assess immune status and predict clinical outcomes of HBV infection. Higher expression levels are associated with a better prognosis. Robek et al., reported IFN- $\lambda 1$ and IFN- $\lambda 2$ inhibited HBV replication [38]. Weekly PEG-IFN- $\lambda$ treatment with or without daily ribavirin for 4 weeks was well tolerated with minimal adverse events and hematologic effects and was associated with clear antiviral activity across a broad range of doses in patients with chronic hepatitis $\mathrm{C}$ virus [39]. Thus, chronic hepatitis B patients with a lower expression of IL-29 should be considered for treatment with IFN- $\lambda 1$ (IL-29). The currents study has two unique features. First, a protein array with a more accurate reflection of secreted cytokines was used to detect a total of 30 cytokines and chemokines associated with antivirus immunity. Simultaneous detection of cytokine expression by array can avoid the error of multiple detection. Second, more stringent inclusion criteria were adopted, such as upper limits of normal levels for ALT [40], HBsAg levels for inactive HBsAg carriers, and HBV DNA levels for immune tolerance patients or inactive HBsAg carriers.

Furthermore, patients that were HBeAg-negative, or with detectable HBV DNA levels, or normal ALT levels were not involved. On one hand, this state is not stable. Activation of hepatitis or elevation of ALT may be present in those with detectable HBV DNA levels. However, the majority of these patients require antivirus treatment.

In summary, for HBV infection patients with persistently normal ALT levels, the up-regulation of cytokines in the JAKSTAT signaling pathway was shown to be an important immune characteristic of HBV infection. The elevation of $\gamma \mathrm{C}$ cytokines, IL-12p70, IL-23p19, and IL-29 in inactive HBV carriers and resolved hepatitis B subjects suggested that changes in immune effects caused by elevation of these cytokines promoted spontaneous $\mathrm{HBeAg}$ seroconversion and HBV clearance.

Acknowledgments The authors wish to thank Prof. Qing Mao and Prof. Guohong Deng for their helpful suggestions, discussions and excellent technical assistance.

Financial Support This work was supported by grants from the Natural Science Foundation of China (30972598 and 81270563) and the State Key Project specialized for HBV-related severe hepatitis of China (2012ZX10002004).

Disclosure The authors declare that they have no conflict of interest.

Open Access This article is distributed under the terms of the Creative Commons Attribution License which permits any use, distribution, and reproduction in any medium, provided the original author(s) and the source are credited.

\section{References}

1. Lok ASF, McMahon BJ. Chronic hepatitis B: Update 2009. Hepatology. 2009;50(3):661-2.

2. European Association For The Study Of The L. EASL clinical practice guidelines: management of chronic hepatitis B virus infection. J Hepatol. 2012;57(1):167-85.

3. Ganem D, Prince AM. Hepatitis B virus infection-natural history and clinical consequences. N Engl J Med. 2004;350(11):1118-29.

4. Rizzetto M, Ciancio A. Chronic HBV-related liver disease. Mol Asp Med. 2008;29(1-2):72-84.

5. Yuen MF, Wong DK, Sablon E, Tse E, Ng IO, Yuan HJ, et al. HBsAg seroclearance in chronic hepatitis $\mathrm{B}$ in the Chinese: virological. Hepatology. 2004;39(6):1694-701.

6. Fattovich G, Giustina G, Sanchez-Tapias J, Quero C, Mas A, Olivotto $\mathrm{PG}$, et al. Delayed clearance of serum HBsAg in compensated cirrhosis B: relation to. Am J Gastroenterol. 1998;93(6):896-900.

7. Chen Y-C, Sheen IS, C-m C, Liaw Y-F. Prognosis following spontaneous HBsAg seroclearance in chronic hepatitis B patients with or without concurrent infection. Gastroenterology. 2002;123(4):1084-9.

8. Arai M, Togo S, Kanda T, Fujiwara K, Imazeki F, Yokosuka O. Quantification of hepatitis B surface antigen can help predict spontaneous. Eur J Gastroenterol Hepatol. 2012;24(4):414-8.

9. Rehermann B, Nascimbeni M. Immunology of hepatitis B virus and hepatitis C virus infection. Nat Rev Immunol. 2005;5(3).

10. Vinader V, Afarinkia K. A beginner's guide to chemokines. Futur Med Chem. 2012;4(7):845-52.

11. Garabedian L, Struyf S, Opdenakker G, Sozzani S, Van Damme J, Laureys G. Langerhans cell histiocytosis: a cytokine/chemokinemediated disorder? Eur Cytokine Netw. 2011;22(3):148-53. 
12. Tacke F, Luedde T, Trautwein C. Inflammatory pathways in liver homeostasis and liver injury. Clin Rev Allergy Immunol. 2009;36(1):4-12.

13. Crispe IN. The liver as a lymphoid organ. Annu Rev Immunol. 2009; 27:147-63.

14. Kowalewska PM, Patrick AL, Fox-Robichaud AE. Innate immunity of the liver microcirculation. Cell Tissue Res. 2011;343(1):85-96.

15. Jensen LJ, Kuhn M, Stark M, Chaffron S, Creevey C, Muller J, et al. STRING 8-a global view on proteins and their functional interactions in 630. Nucleic Acids Res. 2009;37(Database issue):D412-6.

16. Jung MC, Pape GR. Immunology of hepatitis B infection. Lancet Infect Dis. 2002;2(1):43-50.

17. Maini MK, Boni C, Lee CK, Larrubia JR, Reignat S, Ogg GS, et al. The role of virus-specific CD8(+) cells in liver damage and viral control during persistent hepatitis B virus infection. J Exp Med. 2000;191(8):1269-80.

18. Overwijk WW, Schluns KS. Functions of gammaC cytokines in immune homeostasis: current and potential. Clin Immunol. 2009;132(2):153-65.

19. Chen YT, Kung JT. IL-4 inducibility in NKT cells, naive CD4+ T cells and TCR-gamma delta T cells. J Biomed Sci. 2007;14(4):533-8.

20. Hunig T, Luhder F, Elflein K, Gogishvili T, Frohlich M, Guler R, et al. CD28 and IL-4: two heavyweights controlling the balance between immunity and. Med Microbiol Immunol. 2010;199(3):239-46.

21. Gilmour J, Lavender P. Control of IL-4 expression in T helper 1 and 2 cells. Immunology. 2008;124(4):437-44.

22. Villinger F, Miller R, Mori K, Mayne AE, Bostik P, Sundstrom JB, et al. IL-15 is superior to IL-2 in the generation of long-lived antigen specific memory. Vaccine. 2004;22(25-26):3510-21.

23. Yin $\mathrm{W}, \mathrm{Xu} \mathrm{L}$, Sun R, Wei H, Tian Z. Interleukin-15 suppresses hepatitis B virus replication via IFN-beta production. Liver Int. 2012;32(8):1306-14.

24. Parrish-Novak J, Dillon SR, Nelson A, Hammond A, Sprecher C, Gross JA, et al. Interleukin 21 and its receptor are involved in NK cell expansion and regulation. Nature. 2000;408(6808):57-63.

25. Ozaki K, Spolski R, Feng CG, Qi CF, Cheng J, Sher A, et al. A critical role for IL-21 in regulating immunoglobulin production. Science. 2002;298(5598):1630-4.

26. Ma S-W, Huang X, Li Y-Y, Tang L-B, Sun X-F, Jiang X-T, et al. High serum IL-21 levels after 12 weeks of antiviral therapy predict $\mathrm{HBeAg}$ seroconversion in chronic hepatitis B. J Hepatol. 2012;56(4).
27. Mujib S, Jones RB, Lo C, Aidarus N, Clayton K, Sakhdari A, et al. Antigen-independent induction of Tim-3 expression on human $\mathrm{T}$ cells by the common. J Immunol. 2012;188(8):3745-56.

28. Novelli F, Casanova JL. The role of IL-12, IL-23 and IFN-gamma in immunity to viruses. Cytokine Growth Factor Rev. 2004;15(5):367-77.

29. Croxford AL, Mair F, Becher B. IL-23: one cytokine in control of autoimmunity. Eur J Immunol. 2012;42(9):2263-73.

30. Xia L, Tian D, Huang W, Zhu H, Wang J, Zhang Y, et al. Upregulation of IL-23 expression in patients with chronic hepatitis B is mediated. J Immunol. 2012;188(2):753-64.

31. Kao JT, Lai HC, Tsai SM, Lin PC, Chuang PH, Yu CJ, et al. Rather than interleukin-27, interleukin-6 expresses positive correlation with. Liver Int. 2012;32(6):928-36.

32. Hosel M, Quasdorff M, Wiegmann K, Webb D, Zedler U, Broxtermann M, et al. Not interferon, but interleukin- 6 controls early gene expression in hepatitis B. Hepatology. 2009;50(6):1773-82.

33. Uze G, Monneron D. IL-28 and IL-29: newcomers to the interferon family. Biochimie. 2007;89(6-7):729-34.

34. Witte K, Witte E, Sabat R, Wolk K. IL-28A, IL-28B, and IL-29: promising cytokines with type I interferon-like. Cytokine Growth Factor Rev. 2010;21(4):237-51.

35. Gordien E, Rosmorduc O, Peltekian C, Garreau F, Brechot C, Kremsdorf D. Inhibition of hepatitis $\mathrm{B}$ virus replication by the interferon-inducible MxA. J Virol. 2001;75(6):2684-91.

36. Haller O, Staeheli P, Kochs G. Interferon-induced Mx proteins in antiviral host defense. Biochimie. 2007;89(6-7):812-8.

37. Kong XF, Zhang XX, Gong QM, Gao J, Zhang SY, Wang L, et al. $\mathrm{MxA}$ induction may predict sustained virologic responses of chronic hepatitis B patients with IFN-alpha treatment. J Interferon Cytokine Res. 2007;27(9):809-18.

38. Robek MD, Boyd BS, Chisari FV. Lambda interferon inhibits hepatitis B and C virus replication. J Virol. 2005;79(6):3851-4.

39. Muir AJ, Shiffinan ML, Zaman A, Yoffe B, de la Torre A, Flamm S, et al. Phase $1 \mathrm{~b}$ study of Pegylated interferon lambda 1 with or without ribavirin in patients with chronic genotype 1 hepatitis $\mathrm{C}$ virus infection. Hepatology. 2010;52(3):822-32.

40. Prati D, Taioli E, Zanella A, Della Torre E, Butelli S, Del Vecchio E, et al. Updated definitions of healthy ranges for serum alanine aminotransferase levels. Ann Intern Med. 2002;137(1):1-9. 